\title{
DIAGNÓSTICO DE LOS OPERADORES ECONÓMICOS AUTORIZADOS (OEA) EN EL ECUADOR
}

\author{
DIAGNOSIS OF THE AUTHORIZED ECONOMIC OPERATORS (OAS) IN \\ ECUADOR
}

\author{
Pablo Basantes Garcés \\ Universidad Tecnológica Equinoccial \\ pablo.basantes@ute.edu.ec \\ Guido Romero Larco \\ Universidad Tecnológica Equinoccial \\ guido.romero@ute.edu.ec
}

\section{Resumen}

La globalización generó que el ambiente entre las empresas que actúan en los mercados internacionales fuese hiper- competitivo, una mínima diferenciación puede otorgar una ventaja competitiva para cualquiera de los actuantes de la cadena, ya sea por aspectos relacionados con procesos de producción, talento humano, finanzas o marketing. Dentro de este último, en los canales de distribución juega un rol importante la administración de las cadenas logísticas "supply chain managment", donde uno de los factores críticos es su propio aseguramiento.

A partir de los acontecimientos del 11 de septiembre del $2001^{2}$ se potenció la necesidad de mejorar, controlar y asegurar las cadenas logísticas de importadores y exportadores, desde el momento que los productos salen del almacén de despacho, pasan por toda la cadena de operadores de comercio exterior, quienes deben manipular adecuadamente las mercancías además de cumplir con la legislación vigente en los mercados de origen y destino.

Bajo este contexto (OMA, 2005) la Organización Mundial de Aduanas recomendó la implementación de una serie de lineamientos que precisamente salvaguardan la seguridad en la cadena logística, y que se recogen en el denominado Operador Económico Autorizado OEA o AEO (por sus siglas en inglés Authorized Economic Operator), que busca a través de una alianza entre las aduanas de los estados adherentes y el sector privado, la coordinación entre los miembros de la cadena logística para fortalecer su seguridad a cambio de facilitación en los procesos aduaneros. La presente investigación se enfocó en diagnosticar la situación actual del programa de implementación de OEA en el Ecuador, que a la luz de la cantidad de operadores autorizados, se concluye que el programa no ha funcionado, por tanto, fue menester en esta investigación establecer las posibles causas que influyeron para tener estos resultados.

\section{Palabras claves:}

Operador Económico Autorizado OEA, certificación, seguridad, cadena de suministro, sistema de gestión, Acuerdo de Reconocimiento Mutuo ARM

\footnotetext{
1 Luis Luna Osorio (2009) define logística como el conjunto de acciones secuenciales o relacionadas con el flujo de productos, información y pago entre proveedores y consumidores, desde el origen de la materia prima hasta cuando el producto terminado llega al usuario final.

2 Ataque Terrorista a las Torres Gemelas (El Observador 2017)
} 


\begin{abstract}
Globalization generated an atmosphere much more competitive between the companies operating on the international markets. A minimum difference can provide a competitive advantage for any of the acting members of the chain, either by aspects related to processes of production, finance, human resources, or marketing. Within this last inside distribution channels plays an important role the supply chain management, where one of the critical factors is their own assurance.
\end{abstract}

From September 11 attacks, it boosted the need to improve, control and secure the logistic chains of importers and exporters from the moment the products leave the warehouse throughout the whole operators's international trade. Who must properly handling the goods additionally must comply the current legislation in origin and destination markets.

Under this context WCO (WCO, 2005) recommended implementation of a few sets of guidelines to save precisely the security in the supply chain. On behalf the so-called authorized economic operator (AEO) whose seeks through a partnership between Customs authorities in the acceding States and the private sector to coordinate between members of the supply chain in order to strength its security in exchange of facilities in customs processes. This research focused on diagnosing the current situation of the implementation program of OAS in Ecuador that due the number of authorized operators. It concludes that the program did not work therefore, it was necessary in this research to establish possibles causes that influenced to have these results.

\title{
Keywords:
}

Authorized Economic Operator AEO, certification, security, supply chain, management system. ARM Mutual Recognition Agreement.

\section{Metodología}

Nivel de estudio exploratorio, modalidad documental.

El problema analizado no ha sido investigado aún en el contexto ecuatoriano. El propósito de diagnosticar la situación actual de los Operadores Económicos Autorizados OEA en Ecuador fue identificar las probables causas por las cuales a la fecha se han calificado ante el Servicio Nacional de Aduana del Ecuador SENAE un bajo número de operadores de comercio exterior (OCE). Dado que la información de fuentes secundarias existentes sobre el tema es de carácter mundial y que no existen estudios específicos que aborden la temática en cuestión para el Ecuador, por tanto, su alcance fue exploratorio.

\section{Introducción}

El problema de la presente investigación es el mínimo número de Operadores Económicos Autorizados OEA en el Ecuador hasta la presente fecha.

El objetivo general es determinar cuáles han sido las posibles causas por las cuales los operadores de comercio exterior no se han calificado como OEA.

Los objetivos específicos son:

- Revisar los antecedentes a nivel mundial para la existencia de los Operadores Económicos Autorizados OEA. 
- Determinar la situación actual de los Operadores Económicos Autorizados OEA en el Ecuador.

- Establecer las causas por las cuales los operadores de comercio exterior no se han calificado en el Ecuador como Operadores Económicos Autorizados OEA.

La importancia de la logística y de la cadena de suministro gira en torno a la creación de valor para los clientes, proveedores y accionistas. "Este valor se expresa en términos de tiempo y lugar, es decir que los productos tendrán valor en la medida en que estén en manos de los clientes cuando ellos los requieran en el lugar que los demanden" (BALLOU, 2004). La globalización demanda la optimización de los tiempos de traslado y de manejo de los trámites administrativos en origen y destino.

“Adicionalmente, la constante evolución de los medios de transporte y las comunicaciones ha provocado en el mercado homologación de calidad y diseños, poca fidelidad a la marca, proliferación de canales de venta y producto" (Anaya, 2011). La cadena de suministro puede generar ventajas competitivas si se responde a tiempo y con profesionalismo a todas las expectativas del cliente (Rey, 2012), lo cual puede ser decisivo para competir dentro de un mercado. En el proceso de transporte hasta el destino final, uno de los puntos críticos es el control aduanero, las empresas importadoras o exportadoras deberán satisfacer las legislaciones de cada país en el menor tiempo posible. Esta optimización del tiempo se puede dar en la medida en que se efectúen menos acciones de revisión por parte de las autoridades aduaneras de cada país, y solo procede con la cadena logística previamente asegurada.

Además, la gestión de la cadena de suministro puede tener relación directa sobre la optimización en los costos comerciales de las empresas. Según informe de la Organización Mundial de Comercio los costos comerciales de los países en desarrollo equivalen a aplicar al comercio internacional un ad-valoren del $219 \%$ y en los países de ingresos elevados del $134 \%$ (OMC 2015 p.9). La simplificación, armonización, modernización de procedimientos aduaneros y documentación han sido destacados como ejes para lograr una reducción de los costos comerciales (Organización Mundial del Comercio, 2017) lo que podría generar un impulso al comercio mundial de un billón de dólares anuales. (Juan Carlos Amador Hernández, 2017).

La Organización Mundial de Aduanas OMA, organismo técnico internacional, de naturaleza intergubernamental cuya misión es mejorar la eficacia aduanera mundial, en base al Convenio de Kyoto 1974, revisado en 1999 para la armonización de procedimientos aduaneros ha tomado como un factor crítico el uso herramienta tecnologías modernas para facilitar y controlar el comercio legal de bienes, añadiendo un factor adicional que es el de la seguridad de la cadena de suministro en los estados. Para cumplir con este último objetivo en el año 2005 implementó el Marco de Estándares para Facilitar y Asegurar el Comercio SAFE, que constituye la base para el programa de Operador Económico Autorizado OEA.

Por otra parte, dentro del contexto de la seguridad se debe añadir que los gobiernos buscan contrarrestar principalmente dos situaciones que afectan al comercio exterior, la primera los actos criminales y la segunda los actos terroristas (BM, 2009), el 11 de septiembre del 2001 marca un punto de inflexión en la seguridad internacional, cuatro atentados terroristas en Estados Unidos dejan según la Agencia RT daños materiales que ascendieron a los 10.000 millones de dólares, (https://actualidad.rt.com, 2016)este evento repercutió directamente sobre los asuntos relacionados con el comercio internacional, el transporte y la seguridad en la cadena logística.

Bajo estos antecedentes, desde mediados de los años noventa varias han sido las acciones emprendidas, para apoyar la seguridad de las cadenas logísticas comerciales a nivel internacional. A continuación, se pueden detallar las siguientes: 


\section{Normas BASC (Business Alliance for secure commerce- Alianza Empresarial para un comercio seguro)}

De acuerdo a la World Basc Organization WBO, las Normas BASC son un conjunto de estándares establecidos por esta organización desde 1996, que en función de la revisión de los procesos de las empresas involucradas en la cadena de suministro, tienen como objetivo final certificar el sistema de gestión de control y seguridad de la misma. La WBO es una entidad privada cuyo objetivo es crear una cultura de seguridad en el comercio internacional. Los requerimientos exigidos para la certificación BASC apoyan los requisitos solicitados en programas C-TPAT y OEA. El alcance de este programa está direccionado a todas las empresas relacionadas con el comercio internacional, ofreciendo a las empresas asociadas tiempos reducidos de trámites y mejores perfiles de riesgo. (BASC, 2014)

\section{C-TPAT (Certificación voluntaria de la Asociación Aduanera Comercial contra el Terrorismo)}

El gobierno de los Estados Unidos de Norteamérica implementó en el año 2002 este programa para el combate contra el terrorismo.

El C-TPAT nació como una asociación entre gobiernos y el sector privado importador", mediante el cual las empresas obtienen facilidades al ingreso de la mercadería a Estados Unidos de Norteamérica a cambio de llevar a cabo acciones de seguridad en los contenedores y su carga contra posibles acciones terroristas.

Los objetivos que persigue este programa son cuatro: a) Asegurar que los miembros actúen y mejoren la seguridad en la cadena de suministro; b) Otorgar beneficios para las empresas que se certifican; c) Internacionalizar los principios del programa; d) Brindar apoyo a otras iniciativas de la Aduana de Estados Unidos. El principal de los beneficios tiene que ver con la facilitación en los trámites aduaneros que se les otorga a las empresas certificadas, estableciendo tres tipos de categorías a las que puede alcanzar cada miembro. (CEPAL, 2008).

\section{SAFE (Framework of Standars- Marco de Estándares para Facilitar y Asegurar el Comercio)}

En el 2005 la Organización Mundial de Aduanas decidió difundir un Marco de Estándares para Asegurar y Facilitar el Comercio (SAFE), este marco de estándares pretende unir esfuerzos entre las aduanas adscritas a la OMA y los operadores internacionales del sector privado. Sus principales elementos son: 1) la armonización de requisitos de información electrónica avanzada sobre envíos para el interior, exterior o en tránsito, 2) la adopción de un sistema de riesgos para resolver amenazas de seguridad, 3) realización de inspecciones en origen de la carga de alto riesgo a petición de autoridades del país de destino con equipos no intrusivos, y 4) Otorgamiento de beneficios para las empresas que cumplan con la normativa, programa OEA. (OMA, UNECE, 2011)

\section{OEA Unión Europea}

A partir de la difusión del Marco de Estándares para Asegurar y Facilitar el Comercio (SAFE) del año 2005, la Unión Europea estableció la normativa jurídica para implementar las enmiendas de seguridad al Código de Aduanas de la Comunidad (CCC). El programa busca a través de la cooperación entre aduanas y empresas de la comunidad europea fortalecer la seguridad en la cadena de suministro a cambio de beneficios aduaneros en toda la comunidad. El sistema OEA está abierto para cualquiera de los actores de la cadena de suministro y abarca la simplificación aduanera (AEOC), la seguridad y la protección (AEOS) o una combinación de ambos. 
Alrededor de 50 naciones tienen en la actualidad un programa de OEA. que les permite reconocer a sus respectivas aduanas, empresas confiables certificadas de forma mutua por los países de la Unión europea, USA, Canadá, Japón, China, Corea y recientemente por Argentina, Colombia, Costa Rica, Guatemala, México, Panamá, República Dominicana, Uruguay y demás miembros a través de los Acuerdos de Reconocimiento Mutuo (ARM). (COMISION EUROPEA, 2005)

\section{ISO 28000}

La Organización Internacional para la Estandarización ISO es una entidad no gubernamental independiente que cuenta con 162 países afiliados, que se encarga de desarrollar estándares aplicables a nivel mundial para los productos, servicios y sistemas de empresas, que permiten garantizar la calidad, seguridad y eficacia en sus procesos, a través de la consecución de una certificación que avala el cumplimiento de dichos estándares. Dentro de las normas emitidas por ISO encontramos la ISO 28000 implementada en el año 2007, que determina los requerimientos necesarios para implementar en empresas de cualquier tamaño, un sistema de gestión de seguridad enfocado en la cadena de suministro trabaja en las actividades controladas por organizaciones que tienen impacto sobre la cadena. (ISO, 2007)

\section{OEA en Ecuador}

Ecuador es miembro de la Organización Mundial de Aduanas OMA desde el 16 de diciembre de 1997 y desde su adhesión en forma permanente ha implementado sus directrices.

En el año 2010 con la promulgación del Código Orgánico de la Producción e Inversiones COPCI, en el capítulo II denominado De los Operadores Económicos Autorizados, en el artículo 231, el estado ecuatoriano reconoce la existencia del Operador Económico Autorizado OEA, los operadores de comercio exterior que podrían calificarse, y su principal obligación que es el aseguramiento de la cadena logística a cambio de la obtención de facilidades en los trámites aduaneros (COPCI, 2015)

En el caso ecuatoriano la presentación del plan piloto para la implementación del programa OEA, fue realizada el 18 de agosto 2015, en la presentación del proyecto el SENAE hizo énfasis en la meta esperada que tiene que ver con el incremento de las exportaciones y también hacer que otros agentes de comercio exterior se incorporen al programa, importadores, transportistas, agencias de carga y demás eslabones involucrados en la cadena logística (Aulestia, 2017).

En respaldo de esta iniciativa Sergio Mujica, secretario Mundial de Aduanas de la OMA presente en el lanzamiento del programa OEA en Ecuador señalo que "al ser calificado como OEA un exportador obtiene múltiples beneficios: ser más competitivos a nivel mundial, facilitación en trámites aduaneros, reconocimientos de distintas entidades del Estado, además que será una marca diferenciadora que servirá para consolidar y fortalecer un sistema sólido y con prestigio". (MINISTERIO COMERCIO EXTERIOR, 2015)

Posteriormente el SENAE (SENAE, 2015), mediante la Resolución No. SENAE- DGN- 20150720- RE emitida en Guayaquil el 4 de septiembre de 2015, expidió la normativa que permite a los operadores de comercio exterior del Ecuador calificarse como Operador Económico Autorizado OEA. En principio se decidió iniciar como plan piloto la certificación únicamente a los exportadores, seguir luego con los agentes afianzados y posteriormente al resto de los miembros de la cadena logística.

La esencia de dicha normativa radica en que, tanto la aduana del país como la empresa privada pueden aliarse para apoyar la seguridad en la cadena logística de las operaciones de comercio 
internacional, y a su vez, el operador de comercio exterior calificado al amparo de este cuerpo legal podría obtener beneficios que faciliten sus trámites aduaneros al momento de efectuar sus actividades frente al organismo de control en otros países.

\section{"Requisitos para acreditación OEA}

1. Historial Satisfactorio.

2. Sistema de Gestión Administrativa.

3. Adecuada Solvencia Financiera.

4. Estándares Mínimos de Seguridad.

\section{Beneficios:}

\section{Exportadores.}

1. Inclusión de las empresas OEA, en la gestión de riesgos del SENAE.

2. Facilidad y atención prioritaria en los trámites aduaneros.

3. Agilidad en la atención de los trámites de importación que se encuentren directamente relacionados con trámites de exportación.

4. Asignación exclusiva de un especialista OEA del SENAE, para que brinde soporte de forma continua en sus trámites aduaneros.

5. Reconocimiento como un Operador que constantemente implementa medidas de seguridad en sus procesos, para minimizar sus riesgos inmersos en la cadena logística.

6. Publicación de las empresas calificadas como OEA en la página web institucional del SENAE.

7. Mayor Competitividad y prestigio a nivel nacional e internacional.

8. Participación en capacitaciones programadas para las empresas OEA por parte del SENAE.

9. Participación en el Comité Consultivo Público - Privado (se estará a lo dispuesto en la normativa interna).

10. Apertura a nuevos mercados internacionales de manera más ágil, por medio de los Acuerdos de Reconocimiento Mutuo (ARM).

11. Acuerdos de Reconocimiento Mutuo (ARM).

\section{Agentes de Aduana}

1. Reconocimiento como un Operador que constantemente implementa medidas de seguridad en sus procesos, para minimizar sus riesgos inmersos en la cadena logística.

2. Fortalecimiento y consolidación de su presencia en el mercado.

3. Mayor Competitividad y prestigio a nivel nacional e internacional.

4. Asignación exclusiva de un especialista OEA del SENAE, para que brinde soporte en sus trámites concernientes a su licencia de Agente de Aduana.

5. Prioridad en la atención de los trámites concernientes a su licencia de Agente de Aduana.

6. Participación en capacitaciones programadas para las empresas OEA por parte del SENAE

7. Publicación de las empresas calificadas como OEA en la página web institucional del SENAE. 
8. Participación en el Comité Consultivo Público - Privado (se estará a lo dispuesto en la normativa interna)

9. Acuerdos de Reconocimiento Mutuo (ARM).” (https://www.aduana.gob.ec, 2017)

De acuerdo al Compendio del programa Operadores Económicos Autorizados del 2017 publicado por la Organización Mundial de Aduanas, en el período 2015 al 2017 en el Ecuador desde el lanzamiento del proyecto piloto, los Operadores Económicos Autorizados calificados por parte de la Servicio Nacional de Aduana del Ecuador SENAE ascienden a cinco; cuatro operadores son exportadores y uno es agente de aduana (OMA, PERÍODO 2015-2017). Según el portal del SENAE los operadores Económicos Autorizados son:

Tabla 1. Operadores de Comercio Exterior calificados como Operadores Económicos Autorizados en el Ecuador

\begin{tabular}{|c|c|c|c|}
\hline No. & EMPRESA & $\begin{array}{c}\text { TIPO DE } \\
\text { OPERADOR }\end{array}$ & $\begin{array}{l}\text { PROVINCIA } \\
\text { MATRIZ }\end{array}$ \\
\hline 1. & Operadora y Procesadora de Productos Marinos Omarsa & Exportador & Guayas \\
\hline 2. & Acería del Ecuador Adelca & Exportador & Pichincha \\
\hline 3. & Negocios Industriales Real Nirsa & Exportador & Guayas \\
\hline 4. & Pacari & Exportador & Pichincha \\
\hline 5. & Rocalvi & $\begin{array}{ll}\text { Agente } & \text { de } \\
\text { Aduana } & \end{array}$ & Guayas \\
\hline
\end{tabular}

Elaboración: Los autores

Fuente: Portal del SENAE y portal del SRI

Desde noviembre de 2017 el proceso de calificación para la certificación OEA en Ecuador se encuentra suspendido. Con fecha 29 de diciembre de 2017 el SENAE emitió la Resolución Nro. SENAE-SENAE-2017-0693-RE, denominada "Norma Que Regula La Actividad De Los Operadores Económicos Autorizados (OEA)", la cual amplía los requisitos, condiciones, beneficios y obligaciones para los OCE que se califiquen como operador económicos autorizados, sin embargo de lo cual, para la presente investigación el alcance de esta resolución no tiene validez por cuanto el objetivo general es analizar las causas iniciales por las que se obtuvo un bajo número de operadores calificados. El 2 de febrero 2018 mediante Decreto Ejecutivo No. 312 el estado ecuatoriano declara el programa OEA como parte de la Política de Facilitación al Comercio Exterior. Al declararse el programa OEA como Política de Facilitación al Comercio Exterior, el decreto tiene varias repercusiones que se detallan a continuación:

1) Permite al Servicio Nacional de Aduana del Ecuador SENAE gestione Acuerdos de Reconocimiento Mutuo ARM con las aduanas de otros países, inexistentes hasta ese momento.

2) Obliga que otras entidades del estado que participan en el comercio exterior establezcan los mecanismos necesarios para que trabajen en coordinación con la implementación y desarrollo del programa OEA en el Ecuador. Estas entidades son: Ministerio del Interior, Agencia Nacional de Regulación, Control y Vigilancia Sanitaria ARCSA, Agencia Ecuatoriana de Aseguramiento de la Calidad del Agro (Agrocalidad), y Ministerio de Transporte y Obras Públicas.

\section{Reflexiones del proyecto piloto OEA en el Ecuador}

Una vez efectuado el contraste de la información recopilada tanto en fuentes primarias como secundarias se puede plantear las siguientes reflexiones: 
¿Ha funcionado el programa piloto de Operador Económico Autorizado OEA en el Ecuador?

La respuesta de los datos investigados es que apenas cinco operadores de comercio exterior han obtenido la certificación OEA a partir de su aplicación, por lo tanto, el programa piloto ha tenido una baja acogida por parte de los operadores de comercio exterior.

¿Por qué no es atractivo solicitar una calificación como Operador Económico Autorizado OEA para un operador de comercio exterior?

De acuerdo con los datos especificados en la presente investigación, varias son las causas para que no sea interesante:

1. El programa piloto se orientó desde un inicio solo para exportadores y agentes de aduana, quedando por fuera otros operadores calificados por la aduana, importantes en la cadena de suministro tales como: importadores, consolidadoras, agencias de carga, depósitos temporales, depósitos comerciales, operadores portuarios, transportistas e intermediarios. El SENAE presentó el programa OEA a los exportadores debido a que buscó fomentar al sector exportador. Si eso es así, la figura del Agente de Aduana debía excluirse en la presentación del programa OEA al inicio, debido a que para exportar su participación es opcional.

2. Tomando en cuenta que en el año 2014 se encontraban 47,025 (Espin, 2014)) Operadores de Comercio Exterior OCEs registrados, y al año siguiente se puso en práctica el programa OEA, se evidencia en base a los datos de total de acreditaciones OEA hasta la presente, los beneficios no han sido atractivos para los OCEs.

3. Las empresas que obtuvieron su certificación deben retroalimentar al proceso y definir si es complejo cumplir los requisitos solicitados, sin embargo, habría que señalar que cualquier certificación llámese BASC, ISO, C-TPAT, son procesos rigurosos y como tal demandantes más que complicados intrínsecamente.

4. Según las cifras de Proecuador en el año 2016 las exportaciones no petroleras a Estados Unidos de Norteamérica representaron el 22,78\% (Proecuador, 2017) convirtiéndolo en el mayor socio comercial del Ecuador. La certificación C-TPAT, así como la BASC funcionan para este país, bajo este contexto para un operador de comercio exterior ecuatoriano sería más atractivo obtener estas certificaciones.

La política a seguir por parte de la administración aduanera para desarrollar un ARM podría ser el destino de las exportaciones. En nuestro caso nuestro principal socio comercial son los Estados Unidos de Norteamérica y es oportuno desarrollar un ARM con ese país.

5. La certificación OEA nació en la OMA, por tanto, su ámbito de aplicación está circunscrito a la aduana nacional, los acuerdos con las aduanas entre países y el sector privado de los operadores de comercio exterior. Sin embargo, de lo anterior, para que esto funcione de la mejor manera es necesario que también funcionen los Acuerdos de Reconocimiento Mutuo ARM entre países y aduanas, así como también una suerte de coordinación entre entidades estatales del país, que justamente reconozcan la importancia del Operador Económico Autorizado OEA, esto precisamente es lo que persigue el Decreto Ejecutivo 312, puesto que en su parte trascendental al declarar el programa OEA como Política de Facilitación al Comercio Exterior, entre otras acciones establece la coordinación entre entidades del estado, así como la decisión de facultar al SENAE para que gestione los Acuerdo de Reconocimiento Mutuo ARM.

Los ARM buscan razones de ganancia para las partes involucradas, tanto para nuestro país como para su socio en esta materia. En el caso del Ecuador, es obvio que un ARM busca que en el país de destino reconozcan al OEA como tal y por tanto se beneficie de procedimientos 
aduaneros simplificados. La contraparte cuando firma un ARM exigirá la misma reciprocidad, en este caso se refiere a importaciones, por tanto, se debería evaluar la alternativa de incluir al sector importador.

6. La difusión que se le ha otorgado al proyecto piloto de Operador Económico Autorizado OEA, ha venido decreciendo considerablemente, varios pueden ser los factores: el cambio de gobierno que viene con una perspectiva diferente para el Servicio Nacional de Aduana del Ecuador orientada hacia la lucha contra el contrabando (tanto es así que en teoría la tasa de control aduanero tiene ese fin), los bajos resultados alcanzados (cinco operadores calificados en dos años).

Estas reflexiones indican que es necesario plantear un proceso complementario de investigación orientado a satisfacer las interrogantes ¿Por qué no ha funcionado el programa OEA en Ecuador? Y ¿Cuál es la situación actual del programa OEA en los países de la Can? Para lo anterior se plantea efectuar una nueva investigación cuantitativa concluyente, con lo que además se podría recomendar estrategias de potenciación del programa.

\section{Conclusiones}

El programa piloto de Operador Económico Autorizado OEA en Ecuador no ha funcionado como se esperaba ya que a la fecha solo se han calificado cinco operadores de comercio exterior debido a que al momento de su lanzamiento no se contaba con ARM vigentes con los principales socios comerciales del Ecuador.

El SENAE no tomó en cuenta para calificar como OEA al sector más grande de los Operadores de Comercio Exterior, los importadores. Iniciar el plan piloto con el sector exportador limitó la oportunidad de retroalimentación del sector importador que genera recaudación tributaria impositiva y contribuye en la producción nacional y exportaciones.

En el lanzamiento del programa OEA en Ecuador en el año 2015, no existió un plan de implementación para coordinación entre organismos estales relacionados al comercio exterior. El Decreto Ejecutivo 312 del 2 febrero del 2018, determina la interrelación entre organismos del estado como parte de Política de Facilitación al Comercio Exterior.

La certificación de Operador Económico Autorizado contribuye a optimizar tiempos en despacho de aduana, agilizando el comercio y proporcionando ventajas competitivas a empresas importadoras y exportadoras que buscan generar calidad en sus procesos. Las empresas pueden utilizar la certificación OEA para obtener éxito en los mercados internacionales. 


\section{Bibliografía}

Anaya, J. (2011). Madrid: Alfamega.

Aulestia, D. (Intérprete). (18 de Agosto de 2017). Arranca Programa OEA (Operador Ecónomico Autorizado) En Apoyo A Los Exportadores. Quito, Ecuador. Obtenido de http://www.comercioexterior.gob.ec/arranca-programa-oea-operador-economicoautorizado-en-apoyo-a-los-exportadores

BALlOU, R. H. (2004). Logística Administración De La Cadena De Suministro. Mexico: Cámara Nacional de la Industria Editorial Mexicana.

BASC. (2014). World Basc ORganization. Obtenido de www.wbasco.org: https://www.wbasco.org/espanol/documentos/folleto-basc-espanol2014.pdf

BM. (Septiembre de 2009). Banco Mundial. Obtenido de www.bancomundial.org: http://documentos.bancomundial.org/curated/es/862601468339908874/pdf/579700WP0 SCS1G10Box353787B01PUBLIC1.pdf

CEPAL. (2008). Boletín FAL No 258. CEPAL.

COMISION EUROPEA. (2005). https://ec.europa.eu/. Obtenido de https://ec.europa.eu/taxation_customs/general-information-customs/customssecurity/authorised-economic-operator-aeo/authorised-economic-operatoraeo_en\#what_is

COPCI. (5 de Mayo de 2015). Código Orgánico De La Producción, Comercio E Inversiones. Quito: Registro Oficial Organo del Gobierno del Ecuador. Obtenido de https://www.aduana.gob.ec/wp-content/uploads/2018/01/COPCI.pdf

Espin, D. F. (2014). Analísis sobre el impacto del nuevo sistema Ecuapass en los operadores de comercio exterior del Ecuador. Universitario. Obtenido de https://app.ute.edu.ec/sict/Biblioteca/visor/abrir_pedido_digital.aspx?mat_bib_reg=113 $163121002218097117054144177021149161067241094 \&$ mat_bib_dig_cod=107163218 $125254205216196054116130153004044136173 \&$ lectura $=10716321812525420521619$ $6054116130153004044136173 \&$

https://actualidad.rt.com. (11 de 9 de 2016). 11-S: Las preguntas que pocos se atreven a plantear. Obtenido de https://actualidad.rt.com/actualidad/218476-testigos-publicarvideos-11s-atentado-nueva-york,

https://www.aduana.gob.ec. (2017). https://www.aduana.gob.ec. Obtenido de Operador Económico Autorizado - OEA: https://www.aduana.gob.ec/oea/

ISO. (Julio de 2007). Specification for security management systems for the supply chain. Obtenido de https://www.iso.org/standard/44641.html

Juan Carlos Amador Hernández, L. H. (2017). La facilitación del comercio y su aplicación en México. http://revista.ibd.senado.gob.mx/index.php/PluralidadyConsenso/about, 7(34), 29-51. Obtenido de http://revista.ibd.senado.gob.mx/index.php/PluralidadyConsenso/article/view/470 
MINISTERIO COMERCIO EXTERIOR. (4 de septiembre de 2015).

http://www.comercioexterior.gob.ec. Obtenido de

http://www.comercioexterior.gob.ec/arranca-programa-oea-operador-economicoautorizado-en-apoyo-a-los-exportadores/.

OMA. (2005). www.oma.com. Obtenido de http://www.wcoomd.org/en/topics/facilitation/instrument-andtools/tools/ /media/B234A4EA9F224B24961F721D76B419A2.ashx

OMA. (2011). UNECE. Obtenido de http://tfig.unece.org: http://tfig.unece.org/SP/contents/wcosafe.htm

OMA. (PERÍODO 2015-2017). COMPENDIO OEA . OMA.

Organización Mundial del Comercio. (2017). https://www.wto.org/. Obtenido de https://www.wto.org/spanish/forums_s/parliamentarians_s/tfagreefactsheet_s.pdf.

Proecuador. (30 de noviembre de 2017). Resumen de Exportaciones No Petroleras por Destino. Quito, Pichincha, Ecuador. Obtenido de http://www.proecuador.gob.ec/exportadores/publicaciones/monitoreo-de-exportaciones/

Rey, L. (2012). Sistema de Calidad. Buenos Aires: Granica.

SENAE. (2015). Resolución No. SENAE- DGN- 2015- 0720- . Guayaquil: SENAE. 